OPEN ACCESS

Edited by:

Yurong Lai,

Gilead, United States

Reviewed by:

Linsheng Liu,

The First Affiliated Hospital of

Soochow University, China

Stanislav Yanev,

Bulgarian Academy of Sciences

(BAS), Bulgaria

*Correspondence:

Bi-Kui Zhang

505995@csu.edu.cn

Yan-Gang Zhou

zhouyangang@csu.edu.cn

Specialty section:

This article was submitted to

Drug Metabolism and Transport,

a section of the journal

Frontiers in Pharmacology

Received: 18 June 2021

Accepted: 09 August 2021

Published: 25 August 2021

Citation:

Li Y, Deng Y, Zhu Z-Y, Liu Y-P, Xu P, $L i X$, Xie $Y-L$, Yao H-C, Yang L,

Zhang B-K and Zhou Y-G (2021)

Population Pharmacokinetics of Polymyxin B and Dosage Optimization

in Renal Transplant Patients.

Front. Pharmacol. 12:727170.

doi: 10.3389/fphar.2021.727170

\section{Population Pharmacokinetics of Polymyxin B and Dosage Optimization in Renal Transplant Patients}

\author{
Ying $\mathrm{Li}^{1,2,3}$, Yang Deng ${ }^{4}$, Zhen-Yu Zhu ${ }^{1,2}$, Yi-Ping Liu ${ }^{1,2}$, Ping $X u^{1,2}$, Xin $\mathrm{Li}^{4}$, Yue-Liang $\mathrm{Xie}^{5}$, \\ Heng-Chang Yao ${ }^{6}$, Liu Yang ${ }^{7}$, Bi-Kui Zhang ${ }^{1,2,3 *}$ and Yan-Gang Zhou ${ }^{1,2 *}$ \\ ${ }^{1}$ Department of Pharmacy, The Second Xiangya Hospital, Central South University, Changsha, China, ${ }^{2}$ Institute of Clinical \\ Pharmacy, Central South University, Changsha, China, ${ }^{3}$ School of Pharmaceutical Sciences, Central South University, Changsha, \\ China, ${ }^{4}$ Department of Pharmacy, Third Hospital of Changsha, Changsha, China, ${ }^{5}$ Department of Pharmacy, The Third Xiangya \\ Hospital of Central South University, Changsha, China, ${ }^{6}$ Department of Urological Organ Transplantation, The Second Xiangya \\ Hospital, Central South University, Changsha, China, ${ }^{7}$ Hubei Institute of Land Surveying and Mapping, Wuhan, China
}

Currently, polymyxin B has been widely used in the treatment of multidrug-resistant Gramnegative pathogen infections. Due to the limited pharmacokinetic/pharmacodynamic data, the optimal dosage regimen for the recently proposed therapeutic target of the area under the concentration-time curve over $24 \mathrm{~h}$ in steady state divided by the minimum inhibitory concentration 50-100 mg.h/L has not yet been established. Moreover, most studies have focused on critically ill patients, yet there have been no studies in the field of renal transplantation. To optimize the dosage strategy and reduce the risk of toxicity, a population pharmacokinetics model of polymyxin B with the Phoenix NLME program was developed in our study. A total of 151 plasma samples from 50 patients were collected in the present study. Polymyxin B plasma concentrations were measured by highperformance liquid chromatography-tandem mass spectrometry. A one-compartment model adequately described the data, and the clearance and volume of distribution were $1.18 \mathrm{~L} / \mathrm{h}$ and $12.09 \mathrm{~L}$, respectively. A larger creatinine clearance was associated with increased clearance of polymyxin B $(p<0.01)$. Monte Carlo simulation showed that a regimen of a $75 \mathrm{mg}$ loading dose with a $50 \mathrm{mg}$ maintenance dose was a better option to achieve an optimal therapeutic effect (minimum inhibitory concentration $\leq 1 \mathrm{mg} / \mathrm{L}$ ) and to reduce the incidence of side effects for patients with renal impairments. The developed model suggested that dosing adjustment should be based on renal function in renal transplant patients.

Keywords: polymyxin B, PPK (population pharmacokinetics), renal transplant, neurotoxicity, dosing regimens

\section{INTRODUCTION}

Infection due to multidrug-resistant (MDR) Gram-negative bacteria has become an extreme challenge, as MDR organisms have become resistant to most currently available antibiotics, resulting in limited treatment options. Polymyxin B, which was withdrawn from the market due to the high risk of neurotoxicity in the 1970s, has been reused for its high sensitivity against MDR Gram-negative bacteria (Li et al., 2006; Zavascki et al., 2007; Nation et al., 2019).

According to the results from previous pharmacokinetics and pharmacodynamics (PK/PD) studies, the bactericidal activity of polymyxin $\mathrm{B}$ is best correlated with the area under the 
concentration-time curve over $24 \mathrm{~h}$ in steady state divided by the minimum inhibitory concentration $\left(\mathrm{AUC}_{0-24 \mathrm{~h}} / \mathrm{MIC}\right.$ ) (Mohamed et al., 2012). Moreover, the latest guidelines recommended a target plasma $\mathrm{AUC}_{0-24 \mathrm{~h}}$ of $50-100 \mathrm{mg} \cdot \mathrm{h} / \mathrm{L}$ to achieve bactericidal activity against an isolate with an MIC of $2 \mathrm{mg} / \mathrm{L}$ (the EUCAST and CLSI breakpoints) (Tsuji et al., 2019). However, due to the lack of rigorous $\mathrm{PK}$ data, the optimal dosing strategies for polymyxin B remain poorly defined. Previous population PK (PPK) studies of polymyxin B showed significant individual differences across patients (Zavascki et al., 2008; Kwa et al., 2011; Sandri et al., 2013a; Sandri et al., 2013b; Avedissian et al., 2018; Kubin et al., 2018; Manchandani et al., 2018; Miglis et al., 2018; Lakota et al. 2018) found that when given the recommended dosage, only $71 \%$ of patients could achieve the $\mathrm{AUC}_{0-24 \mathrm{~h}}$ target, which might either lead to a poor response or a higher incidence of renal injury (Lakota et al., 2018). Moreover, the significant covariates of polymyxin B pharmacokinetics are controversial. Sandri et al. found that the total body weight was correlated with the clearance rate of polymyxin B, while Miglis et al. found that patients with lower total weight might not be able to achieve $\mathrm{a} \geq 90 \%$ probability of target attainment (PTA) by using weight-based regimens (Sandri et al., 2013b; Miglis et al., 2018). In addition, as polymyxin B was excreted unchanged in urine, some studies found no correlation between polymyxin B clearance (CL) and creatinine clearance (CrCL), whereas Wang et al. found that CrCL was a significant covariable for the CL of polymyxin B (Wang et al., 2020). Further studies are warranted to determine the characteristics of polymyxin B pharmacokinetics.

Due to the frequent use of antimicrobials, prolonged hospitalization and the immune-suppression state, renal transplant recipients are at high risk of MDR Gram-negative infection, which generates an ever-increasing need for polymyxin B employment in their treatment (Kaplan and Meier-Kriesche, 2004; Sayegh and Carpenter, 2004). However, no pharmacokinetics analysis of polymyxin B for patients with kidney transplant has been conducted yet, and the optimal dosage for these patients is not available. It is generally acknowledged that the pharmacokinetics of patients with solid organ transplantations are different from those of normal patients (Han et al., 2010; Chen et al., 2015; Lin et al., 2018). Therefore, the present recommended regimen might not be suitable for renal transplant patients, which might lead to decreased efficacy or a higher risk of toxicity, especially nephrotoxicity. The objectives of our study were to characterize the PPK of polymyxin B in renal transplant patients, to propose dosage regimens of polymyxin $\mathrm{B}$ to achieve the target plasma concentration using Monte Carlo simulation, and to investigate the toxicity of polymyxin B in renal transplant recipients.

\section{MATERIALS AND METHODS}

This prospective study was approved by the Ethics Committee of the Second Xiangya Hospital, Central South University. Informed consent was obtained from all patients or legal representatives of the patients (No. ChiCTR1900022231). Renal transplant patients (aged $\geq 18$ years) who received intravenous polymyxin B (sulfate; Polymyxin B for Injection, China, SPH NO.1 BIOCHEMICAL and PHARMACEUTICAL CO. LTA) for $\geq 48 \mathrm{~h}$ were enrolled. Patients were excluded if they were pregnant, allergic or intolerant to polymyxin $\mathrm{B}$ and lacked the necessary data (weight or some renal function index).

All patients accepted at least 3 days of polymyxin B infusion, and the administration interval of polymyxin $B$ was $12 \mathrm{~h}$ with an infusion duration of 60-120 min. One to six blood samples $(2 \mathrm{~mL})$ were randomly collected $30 \mathrm{~min}$ before the sixth dose of polymyxin B and at $0,0.5,1,2,4,6$, and $8 \mathrm{~h}$ after the end of infusion. Blood samples were centrifuged for $10 \mathrm{~min}(3,400 \mathrm{rpm} /$ $\mathrm{min})$, and the supernatant was immediately stored at $-80^{\circ} \mathrm{C}$ until analysis. A validated high-performance liquid chromatographytandem mass spectrometry (HPLC-MS/MS) was used to measure the concentrations of polymyxin B1 and polymyxin B2 (Thomas et al., 2012). Briefly, a Shim-pack GIST C18 $\left(2.1^{\star} 100 \mathrm{~mm}, 3 \mu \mathrm{m}\right.$, Shimadzu) column was used. Gradient chromatography was performed with solvent A $(0.1 \%$ formic acid in water) and solvent B (acetonitrile). The following gradient elution was performed at a total flow of $0.6 \mathrm{ml} / \mathrm{min}$ for analyte elution: $10-60 \%$ B from 0 to $5 \mathrm{~min}, 60 \%-90 \%$ B from five to $5.10 \mathrm{~min}$, $90 \%$ from 5.10 to $6 \mathrm{~min}, 90 \%-10 \%$ B from six to $6.10 \mathrm{~min}$, and $10 \% \mathrm{~B}$ from 6.10 to $10.0 \mathrm{~min}$. The elution time was $10 \mathrm{~min}$, and the injection volume was $2 \mu \mathrm{L}$. The interday precision was $<12 \%$, the intraday precision was $<9 \%$, and the accuracy ranged from 96.1 to $110.4 \%$. The limit of quantification was $0.03 \mathrm{mg} / \mathrm{L}$.

The following data were collected: age; sex; weight; maintenance doses; time and route of administration; site of infection; microbes; comorbidities; liver function indexes, such as alanine aminotransferase (ALT), aspartate aminotransferase (AST), total bilirubin (TBIL), direct bilirubin (DBIL), and albumin (ALB); and renal function indexes, such as uric acid (UA), blood urea nitrogen (BUN), and CrCL. CrCL was calculated according to the Cockcroft-Gault equation (Janmahasatian et al., 2005). CrCL $\leq 80 \mathrm{ml} / \mathrm{min}$ was defined as renal dysfunction, and when the basic CrCL decreased by more than $25 \%$, nephrotoxicity was defined. Risk, injury, failure, loss and end-stage renal disease criteria were used to define acute kidney injury (AKI) in patients (Bellomo et al., 2004). Neurotoxicity was defined when any neurological symptoms or signs occurred during polymyxin B treatment, including dizziness, vertigo, visual disturbances, confusion, hallucinations, ataxia, seizures, and facial and peripheral paresthesia. The observed $\mathrm{AUC}_{0-24 \mathrm{~h}}$ of patients was calculated by daily dose/CL.

\section{Population Pharmacokinetic Analysis}

The concentration-time data of polymyxin B were analyzed with a nonlinear mixed effect modeling approach with the Phoenix NLME program (version 8.1. Pharsight, A Certara Company, United States of America). The first-order conditional estimation-extended least square method (FOCE-ELS) was used to develop the PPK model. One- and two-compartment models with linear elimination were evaluated for structure model selection based on the objective function value (OFV) 
TABLE 1 | Patients' demographic characteristics.

\begin{tabular}{lc}
\hline Characteristic & Value $^{\mathbf{a}}$ \\
\hline Age (year) & $43.5(18-66)$ \\
Female/Male & $18 / 32$ \\
Actual body weight (kg) & $57.8 \pm 12.4$ \\
Polymyxin B duration (day) & $9(5-52)$ \\
Polymyxin B maintenance dose & \\
50 mg q $12 \mathrm{~h}$ & $20(40.0 \%)$ \\
40 mg q $12 \mathrm{~h}$ & $22(44.0 \%)$ \\
Other doses & $8(16.0 \%)$ \\
Continuous renal replacement & $11(22.0 \%)$ \\
Renal dysfunction & $46(92.0 \%)$ \\
Diabetes mellitus & $7(14.0 \%)$ \\
Hypertension & $28(56.0 \%)$ \\
UA ( $\mu$ mol/L) & $363.0(94.8-752.6)$ \\
ALB (g/L) & $34.7(22.7-49.0)$ \\
BUN (mmol/L) & $20.2(2.65-61.9)$ \\
CrCL (ml/min) & $22.2(4.29-90.7)$ \\
ALT (U/L) & $11.7(4.3-226.7)$ \\
TBIL ( $\mu$ mol/L) & $6.8(2.6-30.6)$ \\
AST (U/L) & $15.7(9.8-76.7)$ \\
DBIL ( $($ mmol/L) & $2.8(1.3-10.3)$ \\
Site of infection & \\
Pulmonary & $45(90.0 \%)$ \\
urinary tract & $4(8.0 \%)$ \\
bloodstream & $1(2.0 \%)$ \\
Microbe & \\
P. aeruginosa & $1(2.0 \%)$ \\
Klebsiella pneumoniae & $3(6.0 \%)$ \\
Escherichia coli & $3(6.0 \%)$ \\
None & $43(86.0 \%)$ \\
\hline & \\
\hline &
\end{tabular}

aValues are the median (range), mean $\pm S D$ or number (\%); UA, uric acid; $A L B$, albumin; $B U N$, blood urea nitrogen; $\mathrm{CrCL}$, creatinine clearance; $A L T$, alanine aminotransferase; TBIL, total bilirubin; AST, aspartate aminotransferase; DBIL, direct bilirubin.

and Akaike information criterion (AIC). The interindividual variability of PK parameters was described by an exponential error model. Residual variability was selected with an additive error model, proportional error model and combined error model. The covariates considered for the modeling included age, weight, ALT, AST, TBIL, DBIL, ALB, BUN, UA, and CrCL. The median of the covariate was used to normalize the covariate, and a stepwise method was used to screen the covariates. A reduction in OFVs of $>3.84(p<0.05)$ was considered to be statistically significant for the inclusion of one additional parameter in the forward inclusion steps, and an increase in OFVs of $>6.63(p<0.01)$ was considered to be statistically significant in the backward elimination steps.

Goodness-of-fit plots were used to assess the validity of the population PK model, which included measured concentrations (DV) versus population prediction (PRED) and individual population prediction (IPRED) plots and conditional weighted residuals (CWRES) versus time and population prediction (PRED) plots. One thousand resample data points were generated by using the bootstrap method to assess the accuracy of the population PK parameters from the final model. The $95 \%$ confidence interval (CI) of each parameter from bootstrapping should encompass the estimates of the final model, and the biases should be less than $10 \%$ with no bias across zero. Moreover, a prediction-corrected visual predictive check (pc-VPC) was used to evaluate the final model performance. A total of 1,000 replicates were calculated based on Monte Carlo simulation, and the $90 \%$ confidence intervals of the fifth, 50th, and 95th percentiles of the simulated concentrations were visually compared with the actual observed data.

\section{Monte Carlo Simulation}

Monte Carlo simulations $(\mathrm{n}=1,000)$ were conducted using metrics to determine the PTA for $f A U_{0-24 h} / M I C \geq 20$ at various MICs $(0.5-2 \mathrm{mg} / \mathrm{L})$ in patients with different renal functions (CrCL: $10-80 \mathrm{ml} / \mathrm{min}$ ), where $f$ is the unbound fraction of polymyxin B (assumed to be 0.42) (Sandri et al., 2013b). The dosages were selected according to the most commonly used regimens in renal transplant patients. The regimens were as follows with a $2 \mathrm{~h}$ infusion: a single $50 \mathrm{mg}$ loading dose followed by either $30 \mathrm{mg}$ every $12 \mathrm{~h}$ or $40 \mathrm{mg}$ every $12 \mathrm{~h}$, either a loading dose of $75 \mathrm{mg}$ or $100 \mathrm{mg}$ followed by $50 \mathrm{mg}$ every $12 \mathrm{~h}$, and a $150 \mathrm{mg}$ loading dose followed by $75 \mathrm{mg}$ every $12 \mathrm{~h}$.

AUCs were calculated for each simulated dosing regimen and each group of renal functions $(\mathrm{CrCL} \leq 30 \mathrm{ml} / \mathrm{min}, 50 \mathrm{ml} / \mathrm{min}$, and $80 \mathrm{ml} / \mathrm{min}$ ) after 24 or $72 \mathrm{~h}$ of therapy to evaluate the probabilities of efficacy and toxicity, respectively. An $\mathrm{AUC}_{0-24 \mathrm{~h}}$ of $50-100 \mathrm{mg} \cdot \mathrm{h} / \mathrm{L}$ was taken as the efficacy exposure of interest according to previous studies (Tsuji et al., 2019).

\section{RESULTS}

\section{Demographic Data}

In total, we collected 151 plasma samples from 50 patients with renal transplant for PK analysis of polymyxin $B$. The demographic characteristics of the patients are summarized in Table 1. The range of CrCL was $4.29-90.7 \mathrm{ml} / \mathrm{min}$, and most patients (92.0\%) were diagnosed with renal insufficiency, of which 29 patients had $\mathrm{CrCL}$ less than $30 \mathrm{ml} / \mathrm{min}$. Eleven patients had continuous renal replacement therapy (CRRT) during the polymyxin $\mathrm{B}$ treatment, and the hemodialysis time was $4 \mathrm{~h}$. Only two patients used loading doses, and the most commonly used maintenance doses were $40 \mathrm{mg}$ and $50 \mathrm{mg} \mathrm{q} 12 \mathrm{~h}$.

Table 2 described how many times of blood samples were taken from each patient, and most of the samples were taken from 39 patients at three different times. Overall, 49 (32.5\%) samples were collected at $0.5 \mathrm{~h}$ before the sixth infusion, and $48(31.8 \%)$ were collected at $0 \mathrm{~h}$ after, the rest were taken at random times

TABLE 2 | Distribution of blood samples.

\section{Number of patients}

Blood samples taken at different times from each patient 
TABLE 3 | Model selection and development

\begin{tabular}{|c|c|c|c|}
\hline Model description & OFV $^{\mathbf{a}}$ & $\Delta \mathrm{OFV}^{\mathrm{b}}$ & $\mathrm{AIC}^{\mathrm{C}}$ \\
\hline One-compartment mode & 465.41 & - & 471.41 \\
\hline Two-compartment model & 465.41 & - & 475.41 \\
\hline \multicolumn{4}{|c|}{ The choice of residual variability model (One-compartment model) } \\
\hline Addictive error model & 402.93 & - & 412.93 \\
\hline Proportional error model & 375.14 & - & 385.14 \\
\hline Combined error model & 375.15 & - & 387.14 \\
\hline Full covariate model & 363.90 & 11.24 & 375.90 \\
\hline
\end{tabular}

${ }^{a}$ objective function value.

${ }^{b}$ change in objective function value in the covariate model.

${ }^{c}$ Akaike information criterion.

during this infusion interval. The plasma concentrations of polymyxin $B$ ranged from $0.44 \mathrm{mg} / \mathrm{L}$ to $8.15 \mathrm{mg} / \mathrm{L}$, and the mean $\mathrm{AUC}_{0-24 \mathrm{~h}}$ was $74.60 \pm 17.81 \mathrm{mg} \cdot \mathrm{h} / \mathrm{L}$.

\section{Development of the PPK Model}

A one-compartment PK model with first-order elimination fit the best in describing the data. The development and selection of the basic model is shown in Table 3. A proportional error model was used to evaluate the residual variability. CrCL was the only significantly effective covariate for the $\mathrm{CL}$ of polymyxin $\mathrm{B}$. No covariate was statistically significant for the volume of distribution (V). The final PK model equations were as follows: CL $(\mathrm{L} / \mathrm{h})=$ $1.18^{\star}(\mathrm{CrCL} / 22.2)^{0.14 *} \exp (\eta \mathrm{CL}) ; \mathrm{V}(\mathrm{L})=12.09^{\star} \exp (\eta \mathrm{V})$. The PPK estimate parameters are presented in Table 4.

The goodness-of-fit plots in the final model are shown in Figure 1. The plots of DV versus IPRED and PRED indicated no structural bias. The plots of CWRES versus PRED and time showed a random distribution around zero, and most of the plasma concentrations were scattered within -2 to 2 , showing that the structure of the final model was not biased and that the model was acceptable.

The estimates of the bootstrap method are presented in Table 4. The estimates of the final model were similar to the median estimates of the bootstrap, and they were within the $95 \%$ CI from the bootstrap analysis, which did not include zero. Additionally, their deviation was less than $10 \%$, indicating that the parameters of the final model were accurate. The result of pc-VPC is shown in Figure 2. Most concentrations were within the $90 \%$ CIs, indicating that the final model had a good description of the original data.

\section{Monte Carlo Simulation}

In the analysis of PTA, all regimens aside from a $50 \mathrm{mg}$ loading dose with $40 \mathrm{mg}$ every $12 \mathrm{~h}$ achieved the target concentration at MIC $\leq 1 \mathrm{mg} / \mathrm{L}$ in all renal function groups on day 3 . Most of the simulated regimens could not achieve adequate target attainment at MICs at the current CLSI and EUCAST breakpoint of $2 \mathrm{mg} / \mathrm{L}$, except $150 \mathrm{mg}$ loading dose with $75 \mathrm{mg}$ maintenance dose in patients with $\mathrm{CrCL} \leq 10 \mathrm{ml} / \mathrm{min}$ (Table 5).

The simulated results of $\mathrm{AUC}_{0-24 \mathrm{~h}}$ at day 1 and day 3 are shown in Table 6. All loading doses made it possible to obtain steady concentrations on day 1 , which was in accordance with the guidelines that loading doses were essential for polymyxin B treatments. However, loading regimens still failed to produce probabilities of efficacious exposures at MICs $\geq 2 \mathrm{mg}$ / L. A single loading dose of $75 \mathrm{mg}$ and a $50 \mathrm{mg}$ maintenance dose was sufficient to achieve the target concentrations on day 1 or day 3 in approximately $90 \%$ of patients.

\section{Adverse Effects}

A total of five patients experienced nephrotoxicity according to our definition (3-11 days), four of whom were classified as risk (3-7 days), and one as injury (11 days). However, different degrees of neurotoxicity were noted in 31 patients (62.0\%): 29 had facial and peripheral paresthesia, three had seizures, and three had dizziness. Seventeen patients (34.0\%) had dermatitis. However, those neurotoxicity symptoms disappeared after polymyxin B withdrawal. Seven patients had pigmentation, and all disappeared 1 month after polymyxin B withdrawal.

\section{DISCUSSION}

This study developed a PPK model of polymyxin B for Chinese renal transplant patients. To the best of our knowledge, this is

TABLE 4 | Population PK parameter estimates in the final model and bootstrap.

\begin{tabular}{|c|c|c|c|c|}
\hline \multirow[t]{2}{*}{ Parameter (unit) } & \multicolumn{2}{|c|}{ Final model results } & \multicolumn{2}{|c|}{ Bootstrap results } \\
\hline & Estimate (shrinkage\%) & $\% \mathrm{CV}^{\mathbf{a}}$ & Median & $95 \% \mathrm{Cl}^{\mathrm{b}}$ \\
\hline$V(L)$ & 12.09 & 6.52 & 11.98 & $10.71-13.67$ \\
\hline$\Theta_{\mathrm{CrCL}}$ & 0.14 & 29.35 & 0.14 & $0.05-0.21$ \\
\hline \multicolumn{5}{|c|}{ Interindividual variability } \\
\hline
\end{tabular}

Residual variability

$\sigma \quad 0.17$




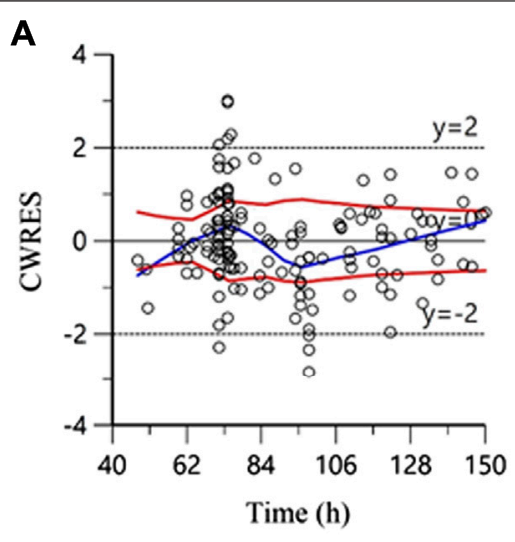

C

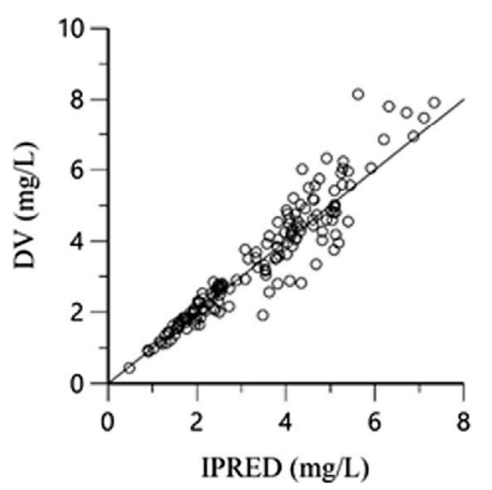

B

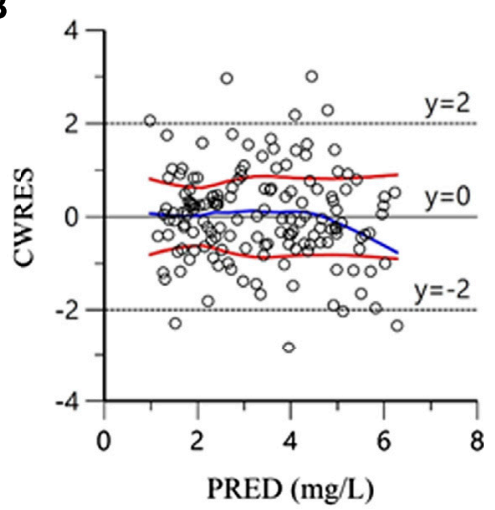

D

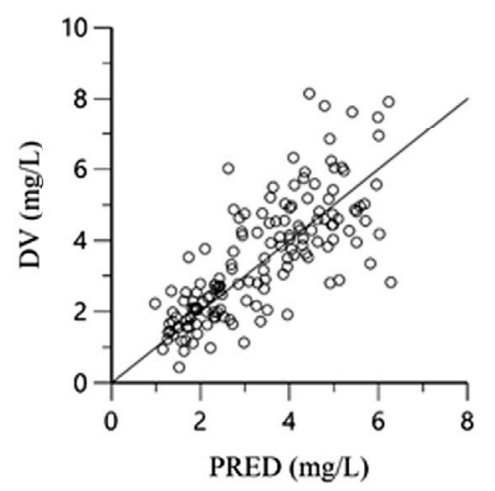

FIGURE 1 | Goodness-of-fit plots for the final model. (A): Conditional weighted residuals versus time; (B): Conditional weighted residuals versus population predicted concentrations; (C): Measured concentrations (DV) versus individual predicted concentrations; (D): Measured concentrations (DV) versus population predicted concentrations.

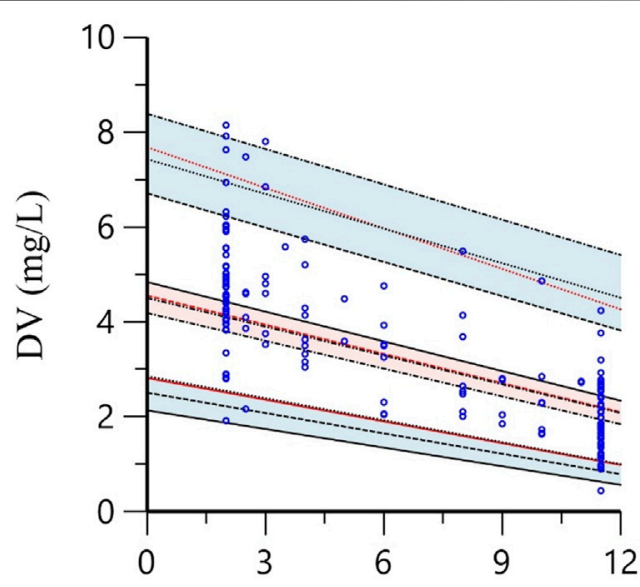

Time after the last dose (h)

FIGURE 2 | The prediction corrected-visual predictive check of the final model. Blue circles represent the observed polymyxin B concentrations. Solid red lines indicate the 5th, 50th and 95th percentiles of the observed data. Black dotted lines show the 5th, 50th and 95th percentiles of the simulated data. The 3 shaded areas represent the $90 \%$ confidence intervals of the 5th, 50th and 95th percentiles of the simulated concentrations. the first study that investigated the pharmacokinetic features and optimized dosage of polymyxin B in renal transplant patients.

In the present study, a one-compartment model was used to describe the original data, which was consistent with some of the previous studies (Kubin et al., 2018; Manchandani et al., 2018). The estimated median value of clearance, $\mathrm{CL}(1.18 \mathrm{~L} / \mathrm{h})$, was lower than the value reported in the previous one-compartment models (CL: 2.37-2.5 L/h) (Kubin et al., 2018; Manchandani et al., 2018). This difference might be mainly due to impaired renal function in the renal transplant patients in this study (CrCL: $20.89 \mathrm{ml} / \mathrm{min}$, range: $4.29-78.84 \mathrm{ml} / \mathrm{min}$ ) because we identified $\mathrm{CrCL}$ as a significant predictor of $\mathrm{CL}$ in the covariate analysis. After including the covariate of $\mathrm{CrCL}$ in the model, the interindividual variability of CL was reduced from 28.11 to $24.49 \%$ in the final PPK model. It has been debated whether CrCL has a significant effect on the CL of polymyxin B. Sandri et al. found that polymyxin $B$ is not significantly eliminated by the kidneys, and some other PPK studies demonstrated that polymyxin B clearance does not depend on CrCL (Sandri et al., 2013b; Kubin et al., 2018; Miglis et al., 2018). According to these results, no dose adjustment was recommended by the international consensus guidelines (Tsuji et al., 2019). However, 
TABLE 5 | PTA based on different CrCL and doses administration on day 3.

\begin{tabular}{|c|c|c|c|c|c|c|}
\hline \multirow{2}{*}{$\begin{array}{c}\mathrm{CrCL} \\
(\mathrm{ml} / \mathrm{min})\end{array}$} & \multirow{2}{*}{$\begin{array}{l}\mathrm{MIC}^{\mathrm{a}} \\
(\mathrm{mg} / \mathrm{l})\end{array}$} & \multicolumn{5}{|c|}{ Dosage $^{b}$ of PTA (mg) } \\
\hline & & $50+30$ & $50+40$ & $75+50$ & $100+50$ & $150+75$ \\
\hline \multirow{3}{*}{10} & 0.5 & 1 & 1 & 1 & 1 & 1 \\
\hline & 1 & 0.83 & 1 & 1 & 1 & 1 \\
\hline & 2 & 0.02 & 0.10 & 0.38 & 0.39 & 0.92 \\
\hline \multirow{3}{*}{20} & 0.5 & 1 & 1 & 1 & 1 & 1 \\
\hline & 1 & 0.72 & 1 & 1 & 1 & 1 \\
\hline & 2 & 0.01 & 0.04 & 0.25 & 0.26 & 0.85 \\
\hline \multirow{3}{*}{30} & 0.5 & 1 & 1 & 1 & 1 & 1 \\
\hline & 1 & 0.64 & 1 & 1 & 1 & 1 \\
\hline & 2 & 0 & 0.03 & 0.18 & 0.19 & 0.79 \\
\hline \multirow{3}{*}{40} & 0.5 & 1 & 1 & 1 & 1 & 1 \\
\hline & 1 & 0.57 & 1 & 1 & 1 & 1 \\
\hline & 2 & 0 & 0.02 & 0.15 & 0.15 & 0.74 \\
\hline \multirow{3}{*}{50} & 0.5 & 1 & 1 & 1 & 1 & 1 \\
\hline & 1 & 0.53 & 1 & 1 & 1 & 1 \\
\hline & 2 & 0 & 0.02 & 0.12 & 0.13 & 0.70 \\
\hline \multirow{3}{*}{60} & 0.5 & 1 & 1 & 1 & 1 & 1 \\
\hline & 1 & 0.48 & 1 & 1 & 1 & 1 \\
\hline & 2 & 0 & 0.01 & 0.10 & 0.11 & 0.67 \\
\hline \multirow{3}{*}{70} & 0.5 & 1 & 1 & 1 & 1 & 1 \\
\hline & 1 & 0.45 & 1 & 1 & 1 & 1 \\
\hline & 2 & 0 & 0.01 & 0.09 & 0.09 & 0.63 \\
\hline \multirow{3}{*}{80} & 0.5 & 1 & 1 & 1 & 1 & 1 \\
\hline & 1 & 0.43 & 1 & 1 & 1 & 1 \\
\hline & 2 & 0 & 0.01 & 0.07 & 0.08 & 0.58 \\
\hline
\end{tabular}

${ }^{a}$ minimum inhibitory concentration.

${ }^{b}$ loading dose + maintenance dose twice daily; CrCL, creatinine clearance; MIC: minimum inhibitory concentration; PTA, probability of target attainment; gray background: groups that have not reached the target PTA\%.

urinary recovery varied considerably between different patients in a previous study (range, $0.98-17.4 \%$ ), which indicated that the renal excretion of polymyxin $B$ in some patients might not be very low (Sandri et al., 2013b). Moreover, Manchandani et al. found that $\mathrm{CrCL}$ was a significant covariate for $\mathrm{CL}$ of polymyxin $\mathrm{B}$, and the CL value in renal dysfunction patients was found to be lower than (marginal significance) that in patients without renal impairment according to Thamlikitkul's results (Thamlikitkul et al., 2017; Manchandani et al., 2018). With larger samples and more severe renal impairment, the influence of $\mathrm{CrCL}$ on polymyxin B clearance seemed to be more significant in our study, but larger PK studies in patients with renal insufficiency are needed to validate the relationship between renal function and polymyxin B clearance.

We also assessed the relationship between total body weight and polymyxin B CL. Similar to other studies, a general lack of a significant linear relationship between weight and polymyxin B clearance was found in the renal transplant patient population (Kubin et al., 2018; Miglis et al., 2018; Wang et al., 2020). In addition, a previous Monte Carlo simulation found that in the weight-based dosing scheme, patients in the lowest body weight strata were far from achieving $>90 \%$ PTA, while patients in the highest body weight strata had a higher incidence of toxic exposures (Miglis et al., 2018). Future work should quantify the full relationship between weight and polymyxin B clearance and whether any one weight-based dosing scheme should be used.

The mean V $(12.09 \mathrm{~L})$ in our study was also lower than the data reported by previous studies (V: 34.3-34.4 L) (Kubin et al., 2018; Manchandani et al., 2018), which indicates that polymyxin $\mathrm{B}$ might have less tissue distribution in renal transplant patients. Polymyxin B is mainly distributed in the kidney, and the renal tissue/serum concentration ratio of polymyxin B was 19.62 at $6 \mathrm{~h}$ after dosing in rats (Manchandani et al., 2015). Therefore, the variation in polymyxin $B$ accumulation in the kidney might be responsible for the differences in the apparent volume of distribution. In addition to the exceptional pharmacokinetic characteristics of renal transplant patients, most of our patients had severe renal impairment. Therefore, decreased glomerular 
TABLE 6 | $\mathrm{AUC}_{0-24 \mathrm{~h}}$ based on different $\mathrm{CrCL}$ and doses administration on day 1 and day 3.

\begin{tabular}{|c|c|c|c|c|c|c|c|c|c|}
\hline \multirow{2}{*}{ DAY } & \multicolumn{3}{|c|}{$\leq 30 \mathrm{ml} / \mathrm{min}$} & \multicolumn{3}{|c|}{$\leq 50 \mathrm{ml} / \mathrm{min}$} & \multicolumn{3}{|c|}{$\leq 80 \mathrm{ml} / \mathrm{min}$} \\
\hline & $\mathrm{P} 10$ & P50 & $\mathrm{P} 90$ & $\mathrm{P} 10$ & P50 & P90 & $\mathrm{P} 10$ & P50 & P90 \\
\hline \multicolumn{10}{|c|}{ 50-mg loading dose with $30-\mathrm{mg}$ maintenance dose twice daily } \\
\hline Day 1 & 39.73 & 51.6 & 65.64 & 37.74 & 49.37 & 63.15 & 35.9 & 47.27 & 60.74 \\
\hline Day 3 & 34.37 & 46.18 & 63.67 & 32.11 & 43.26 & 59.85 & 30.18 & 40.65 & 56.43 \\
\hline \multicolumn{10}{|c|}{ 50-mg loading dose with 40-mg maintenance dose twice daily } \\
\hline Day 1 & 43.29 & 56.75 & 71.78 & 41.03 & 54.24 & 68.76 & 39.11 & 51.93 & 66.02 \\
\hline Day 3 & 45.57 & 61.27 & 83.15 & 42.60 & 57.30 & 77.92 & 40.07 & 53.92 & 73.42 \\
\hline \multicolumn{10}{|c|}{ 75-mg loading dose with 50-mg maintenance dose twice daily } \\
\hline Day 1 & 61.56 & 79.9 & 101.1 & 56.3 & 75.88 & 96.75 & 55.62 & 72.94 & 92.92 \\
\hline Day 3 & 56.97 & 76.72 & 104.44 & 53.26 & 71.73 & 97.78 & 50.07 & 67.43 & 91.99 \\
\hline \multicolumn{10}{|c|}{ 100-mg loading dose with 50-mg maintenance dose twice daily } \\
\hline Day 1 & 75.51 & 97.73 & 123.83 & 71.71 & 93.22 & 118.47 & 68.41 & 89.08 & 113.6 \\
\hline Day 3 & 57.02 & 76.97 & 105.29 & 53.29 & 71.91 & 98.57 & 50.08 & 67.57 & 92.60 \\
\hline \multicolumn{10}{|c|}{ 150-mg loading dose with $75-\mathrm{mg}$ maintenance dose twice daily } \\
\hline Day 1 & 113.26 & 146.59 & 185.74 & 104.15 & 139.12 & 177.55 & 102.62 & 133.61 & 170.41 \\
\hline Day 3 & 85.65 & 115.66 & 158.32 & 79.94 & 107.87 & 147.85 & 71.57 & 100.47 & 138.51 \\
\hline
\end{tabular}

${ }_{A \cup C_{0-24 h}}$ area under the concentration-time curve over $24 \mathrm{~h}$; CrCL: creatinine clearance; $P 10,10^{\text {th }}$ percentile; $P 50,50^{\text {th }}$ percentile; $P 90,90^{\text {th }}$ percentile.

filtration might reduce the distribution of polymyxin $\mathrm{B}$ in the kidney in these patients, which leads to a lower $\mathrm{V}$ value. This speculation that patients with renal impairment might have lower renal exposure to polymyxin $\mathrm{B}$ is also consistent with a previous study, which found that when both groups were given high-dose polymyxin $\mathrm{B}$, patients with $\mathrm{CrCL} \geq 90 \mathrm{ml} / \mathrm{min}$ were more likely to develop AKI than patients with mild renal impairment (John et al., 2017). These results highlighted the need for further investigation to better define the distribution of polymyxin $\mathrm{B}$.

This is the first study to simulate the PTA and $\mathrm{AUC}_{0-24 \mathrm{~h}}$ of different regimens in patients based on renal function. Our results showed that the regimen of a 50-mg loading dose with a 40-mg maintenance dose was sufficient to reach the target PTA with an MIC $\leq 1 \mathrm{mg} / \mathrm{L}$ for patients with renal dysfunction (CrCL $\leq 80 \mathrm{ml} /$ $\mathrm{min}$ ), which was much lower than the dosage recommended by other studies (Miglis et al., 2018; Tsuji et al., 2019). This might be due to the unique PPK features of polymyxin B in renal transplant patients, which was in accordance with previous studies. Xiao-bin et al. found that the voriconazole CL in patients with renal transplant was similar to the result of patients with lung transplant, which was much lower than that in normal patients (Lin et al., 2018). However, most of the simulated regimens failed to achieve adequate target attainment at an MIC of $2 \mathrm{mg} / \mathrm{L}$, which suggests that patients might experience poor clinical outcome when treated with polymyxin $\mathrm{B}$ in such circumstances.

$\mathrm{AUC}_{0-24 \mathrm{~h}}$ of regimens of $\geq 50 \mathrm{mg}$ maintenance dose all achieved the proposed therapeutic target of $50-100 \mathrm{mg} \cdot \mathrm{h} / \mathrm{L}$ on day 3 , and with a loading dose of $100 \mathrm{mg}$, the target concentration can be quickly achieved on the first day, which is more prominent for critically ill patients. However, polymyxin B seemed tolerated poorly in the renal transplant patients due to the neurotoxicity, thus, the determination of optimal dosing strategies should be based on the balance between the toxicity and the need for early efficacious exposures.

It is well known that nephrotoxicity is the major adverse effect of polymyxin $\mathrm{B}$, with a wide range from 26.9 to $60 \%$ (Plachouras et al., 2009; Akajagbor et al., 2013; Dubrovskaya et al., 2015; Rigatto et al., 2015). In the present study, however, the incidence of AKI was extremely low, with only five patients showing nephrotoxicity. As polymyxin B-induced nephrotoxicity is a dose-limiting adverse effect, our result seemed to be consistent with the previous finding that patients with renal impairment might have a lower incidence of AKI due to the decreased percentage of tubular reabsorption with $\mathrm{CrCL}$ and thus the lower renal exposure to polymyxin B. In addition, older age has been found to be a risk factor for polymyxin $\mathrm{B}$-induced AKI, and the lower risk of AKI in our patients might also be due to the younger average age (Rigatto et al., 2015).

The incidence of neurotoxicity was much higher than noted before (62.0 vs 7\%) and has become the dose-limiting factor in our study (Arnold et al., 2007). The mechanism of polymyxin B-induced neurotoxicity is not yet clear, and some studies have demonstrated that colistin-induced neurotoxicity is dose-dependent and significantly associated with the apoptosis of neuronal cells (Lu et al., 2017a; Lu et al., 2017b; Dai et al., 2017; Dai et al., 2018a; Dai et al., 2018b). Considering their similar structures, this mechanism 
might also fit polymyxin B; therefore, the higher exposure of polymyxin $B$ in renal transplant patients might induce more severe neurotoxicity. In addition, the immunosuppressive agents taken by our patients, such as tacrolimus and cyclosporine, can also induce neurotoxicity (Eidelman et al., 1991; Wijdicks et al., 1994; Erer et al., 1996), and this combination might increase the incidence of this adverse effect.

There are several limitations in the present study. First, the sample size was small, which resulted in the limited analysis of risk factors for neurotoxicity. Second, all of our patients had renal dysfunction, and we could not investigate the PKs of renal transplant patients with normal renal function.

To our knowledge, this is the first population PK model and CrCL-based simulation established in renal transplant patients. Our results suggested that renal function might have a significant effect on the clearance of polymyxin B, and an adjusted dosage regime might be needed in patients with renal impairments. Further investigation of the relationships between polymyxin B pharmacokinetics and renal function is urgently needed.

\section{DATA AVAILABILITY STATEMENT}

The original contributions presented in the study are included in the article/supplementary material, further inquiries can be directed to the corresponding authors.

\section{REFERENCES}

Akajagbor, D. S., Wilson, S. L., Shere-Wolfe, K. D., Dakum, P., Charurat, M. E., and Gilliam, B. L. (2013). Higher Incidence of Acute Kidney Injury with Intravenous Colistimethate Sodium Compared with Polymyxin B in Critically Ill Patients at a Tertiary Care Medical center. Clin. Infect. Dis. 57, 1300-1303. doi:10.1093/cid/cit453

Arnold, T. M., Forrest, G. N., and Messmer, K. J. (2007). Polymyxin Antibiotics for Gram-Negative Infections. Am. J. Health Syst. Pharm. 64 (8), 819-826. doi:10.2146/ajhp060473

Avedissian, S., Miglis, C., Kubin, C. J., Rhodes, N. J., Yin, M. T., Cremers, S., et al. (2018). Polymyxin B Pharmacokinetics in Adult Cystic Fibrosis Patients. Pharmacotherapy 38 (7), 730-738. doi:10.1002/phar.2129

Bellomo, R., Ronco, C., Kellum, J. A., Mehta, R. L., and Palevsky, P. (2004). Acute Renal Failure - Definition, Outcome Measures, Animal Models, Fluid Therapy and Information Technology Needs: The Second International Consensus Conference of the Acute Dialysis Quality Initiative (ADQI) Group. Crit. Care 8, R204-R212. doi:10.1186/cc2872

Chen, W., Xie, H., Liang, F., Meng, D., Rui, J., Yin, X., et al. (2015). Population Pharmacokinetics in China: the Dynamics of Intravenous Voriconazole in Critically Ill Patients with Pulmonary Disease. Biol. Pharm. Bull. 38, 996-1004. doi:10.1248/bpb.b14-00768

Dai, C., Ciccotosto, G. D., Cappai, R., Tang, S., Li, D., Xie, S., et al. (2018). Curcumin Attenuates Colistin-Induced Neurotoxicity in N2a Cells via AntiInflammatory Activity, Suppression of Oxidative Stress, and Apoptosis. Mol. Neurobiol. 55 (1), 421-434. doi:10.1007/s12035-016-0276-6

Dai, C., Ciccotosto, G. D., Cappai, R., Wang, Y., Tang, S., Hoyer, D., et al. (2018). Rapamycin Confers Neuroprotection against Colistin-Induced Oxidative Stress, Mitochondria Dysfunction, and Apoptosis through the Activation of Autophagy and mTOR/Akt/CREB Signaling Pathways. ACS Chem. Neurosci. 9 (4), 824-837. doi:10.1021/acschemneuro.7b00323

Dai, C., Ciccotosto, G. D., Cappai, R., Wang, Y., Tang, S., Xiao, X., et al. (2017). Minocycline Attenuates Colistin-Induced Neurotoxicity via Suppression of

\section{ETHICS STATEMENT}

The studies involving human participants were reviewed and approved by the Chang SHU+ the Second Xiangya Hospital, Central South University; Jing-ping LIU + the Second Xiangya Hospital, Central South University; Luo-sheng TANG+ the Second Xiangya Hospital, Central South University; Guang-zhi ZHOU+ the Second Xiangya Hospital, Central South University; Jiang-dong NI+ the Second Xiangya Hospital, Central South University. The patients/participants provided their written informed consent to participate in this study.

\section{AUTHOR CONTRIBUTIONS}

YL wrote the manuscript; B-KZ and Y-GZ designed the research; YD and Z-YZ performed the research; Y-LX, PX and LY analyzed the data; XL, H-CY and Y-PL contributed new reagents/analytical tools.

\section{FUNDING}

Grants from the Department of Science and Technology of Hunan Province (No. 2017SK50119), the Project of Health and Health Commission of Hunan Province, China (No. 20201375), and The Natural Science Foundation of Hunan Province, China (No. 2019JJ50680), were used to support this research.

Apoptosis, Mitochondrial Dysfunction and Oxidative Stress. J. Antimicrob. Chemother. 72 (6), 1635-1645. doi:10.1093/jac/dkx037

Dubrovskaya, Y., Prasad, N., Lee, Y., Esaian, D., Figueroa, D. A., and Tam, V. H. (2015). Risk Factors for Nephrotoxicity Onset Associated with Polymyxin B Therapy. J. Antimicrob. Chemother. 70, 1903-1907. doi:10.1093/jac/dkv014

Eidelman, B. H., Abu-Elmagd, K., Wilson, J., Fung, J. J., Alessiani, M., Jain, A., et al. (1991). Neurologic Complications of FK 506. Transpl. Proc. 23, 3175-3178.

Erer, B., Polchi, P., Lucarelli, G., Angelucci, E., Baronciani, D., Galimberti, M., et al. (1996). CsA-Associated Neurotoxicity and Ineffective Prophylaxis with Clonazepam in Patients Transplanted for Thalassemia Major: Analysis of Risk Factors. Bone Marrow Transpl. 18, 157-162.

Han, K., Capitano, B., Bies, R., Potoski, B. A., Husain, S., Gilbert, S., et al. (2010). Bioavailability and Population Pharmacokinetics of Voriconazole in Lung Transplant Recipients. Antimicrob. Agents Chemother. 54, 4424-4431. doi:10.1128/AAC.00504-10

Janmahasatian, S., Duffull, S. B., Ash, S., Ward, L. C., Byrne, N. M., and Green, B. (2005). Quantification of Lean Bodyweight. Clin. Pharmacokinet. 44, 1051-1065. doi:10.2165/00003088-200544100-00004

John, J. F., Falci, D. R., Rigatto, M. H., Oliveira, R. D., Kremer, T. G., and Zavascki, A. P. (2017). Severe Infusion-Related Adverse Events and Renal Failure in Patients Receiving High-Dose Intravenous Polymyxin B. Antimicrob. Agents Chemother. 62 (1), e01617-17. doi:10.1128/AAC.01617-17

Kaplan, B., and Meier-Kriesche, H. U. (2004). Renal Transplantation: A Half century of success and the Long Road Ahead. J. Am. Soc. Nephrol. 15 (12), 3270-3271. doi:10.1097/01.ASN.0000146569.59482.8C

Kubin, C. J., Nelson, B. C., Miglis, C., Scheetz, M. H., Rhodes, N. J., Avedissian, S. N., et al. (2018). Population Pharmacokinetics of Intravenous Polymyxin B from Clinical Samples. Antimicrob. Agents Chemother. 62 (3), e01493-17. doi:10.1128/AAC.01493-17

Kwa, A. L., Abdelraouf, K., Low, J. G., and Tam, V. H. (2011). Pharmacokinetics of Polymyxin B in a Patient with Renal Insufficiency: A Case Report. Clin. Infect. Dis. 52 (10), 1280-1281. doi:10.1093/cid/cir137

Lakota, E. A., Landersdorfer, C. B., Nation, R. L., Li, J., Kaye, K. S., Rao, G. G., et al. (2018). Personalizing Polymyxin B Dosing Using an Adaptive Feedback 
Control Algorithm. Antimicrob. Agents Chemother. 62 (7), e00483-18. doi:10.1128/AAC.00483-18

Li, J., Nation, R. L., Turnidge, J. D., Milne, R. W., Coulthard, K., Rayner, C. R., et al. (2006). Colistin: the Re-emerging Antibiotic for Multidrug-Resistant Gram-Negative Bacterial Infections. Lancet Infect. Dis. 6 (9), 589-601. doi:10.1016/S1473-3099(06)70580-1

Lin, X. B., Li, Z. W., Yan, M., Zhang, B. K., Liang, W., Wang, F., et al. (2018). Population Pharmacokinetics of Voriconazole and CYP2C19 Polymorphisms for Optimizing Dosing Regimens in Renal Transplant Recipients. Br. J. Clin. Pharmacol. 84, 1587-1597. doi:10.1111/bcp.13595

Lu, Z., Chen, C., Wu, Z., Miao, Y., Muhammad, I., Ding, L., et al. (2017). A Dual Role of P53 in Regulating Colistin-Induced Autophagy in PC-12 Cells. Front. Pharmacol. 8, 768. doi:10.3389/fphar.2017.00768

Lu, Z., Jiang, G., Chen, Y., Wang, J., Muhammad, I., Zhang, L., et al. (2017). Salidroside Attenuates Colistin-Induced Neurotoxicity in RSC96 Schwann Cells through PI3K/ Akt Pathway. Chem. Biol. Interact 271, 67-78. doi:10.1016/j.cbi.2017.04.027

Manchandani, P., Thamlikitkul, V., Dubrovskaya, Y., Babic, J. T., Lye, D. C., Lee, L. S., et al. (2018). Population Pharmacokinetics of Polymyxin B. Clin. Pharmacol. Ther. 104 (3), 534-538. doi:10.1002/cpt.981

Manchandani, P., Zhou, J., Ledesma, K. R., Truong, L. D., Chow, D. S., Eriksen, J. L., et al. (2015). Characterization of Polymyxin B Biodistribution and Disposition in an Animal Model. Antimicrob. Agents Chemother. 60, 1029-1034. doi:10.1128/AAC.02445-15

Miglis, C., Rhodes, N. J., Avedissian, S. N., Kubin, C. J., Yin, M. T., Nelson, B. C., et al. (2018). Population Pharmacokinetics of Polymyxin B in Acutely Ill Adult Patients. Antimicrob. Agents Chemother. 62 (3), e01475-17. doi:10.1128/ AAC.01475-17

Mohamed, A. F., Karaiskos, I., Plachouras, D., Karvanen, M., Pontikis, K., Jansson, B., et al. (2012). Application of a Loading Dose of Colistin Methanesulfonate in Critically Ill Patients: Population Pharmacokinetics, Protein Binding, and Prediction of Bacterial Kill. Antimicrob. Agents Chemother. 56, 4241-4249. doi:10.1128/AAC.06426-11

Nation, R. L., Rigatto, M. H. P., Falci, D. R., and Zavascki, A. P. (2019). Polymyxin Acute Kidney Injury: Dosing and Other Strategies to Reduce Toxicity. Antibiotics (Basel) 8 (1), 24. doi:10.3390/antibiotics8010024

Plachouras, D., Karvanen, M., Friberg, L. E., Papadomichelakis, E., Antoniadou, A., Tsangaris, I., et al. (2009). Population Pharmacokinetic Analysis of Colistin Methanesulfonate and Colistin after Intravenous Administration in Critically Ill Patients with Infections Caused by Gram-Negative Bacteria. Antimicrob. Agents Chemother. 53, 3430-3436. doi:10.1128/AAC.01361-08

Rigatto, M. H., Behle, T. F., Falci, D. R., Freitas, T., Lopes, N. T., Nunes, M., et al. (2015). Risk Factors for Acute Kidney Injury (AKI) in Patients Treated with Polymyxin B and Influence of AKI on Mortality: A Multicentre Prospective Cohort Study. J. Antimicrob. Chemother. 70 (5), 1552-1557. doi:10.1093/jac/dku561

Sandri, A. M., Landersdorfer, C. B., Jacob, J., Boniatti, M. M., Dalarosa, M. G., Falci, D. R., et al. (2013). Population Pharmacokinetics of Intravenous Polymyxin B in Critically Ill Patients: Implications for Selection of Dosage Regimens. Clin. Infect. Dis. 57 (4), 524-531. doi:10.1093/cid/cit334

Sandri, A. M., Landersdorfer, C. B., Jacob, J., Boniatti, M. M., Dalarosa, M. G., Falci, D. R., et al. (2013). Pharmacokinetics of Polymyxin B in Patients on Continuous Venovenous Haemodialysis. J. Antimicrob. Chemother. 68 (3), 674-677. doi:10.1093/jac/dks437
Sayegh, M. H., and Carpenter, C. B. (2004). Transplantation 50 Years LaterPprogress, Challenges, and Promises. N. Engl. J. Med. 351 (26), 2761-2766. doi:10.1056/NEJMon043418

Thamlikitkul, V., Dubrovskaya, Y., Manchandani, P., Ngamprasertchai, T., Boonyasiri, A., Babic, J. T., et al. (2017). Dosing and Pharmacokinetics of Polymyxin B in Patients with Renal Insufficiency. Antimicrob. Agents Chemother. 61, e01337-e01316. doi:10.1128/AAC.01337-16

Thomas, T. A., Broun, E. C., Abildskov, K. M., Kubin, C. J., Horan, J., Yin, M. T., et al. (2012). High Performance Liquid Chromatography-Mass Spectrometry Assay for Polymyxin B1 and B2 in Human Plasma. Ther. Drug Monit. 34, 398-405. doi:10.1097/FTD.0b013e31825c827a

Tsuji, B. T., Pogue, J. M., Zavascki, A. P., Paul, M., Daikos, G. L., Forrest, A., et al. (2019). International Consensus Guidelines for the Optimal Use of the Polymyxins: Endorsed by the American College of Clinical Pharmacy (ACCP), European Society of Clinical Microbiology and Infectious Diseases (ESCMID), Infectious Diseases Society of America (IDSA), International Society for Anti-Infective Pharmacology (ISAP), Society of Critical Care Medicine (SCCM), and Society of Infectious Diseases Pharmacists (SIDP). Pharmacotherapy 39, 10-39. doi:10.1002/phar.2209

Wang, P., Zhang, Q., Zhu, Z., Feng, M., Sun, T., Yang, J., et al. (2020). Population Pharmacokinetics and Limited Sampling Strategy for Therapeutic Drug Monitoring of Polymyxin B in Chinese Patients with Multidrug-Resistant Gram-Negative Bacterial Infections. Front. Pharmacol. 11, 829. doi:10.3389/ fphar.2020.00829

Wijdicks, E. F., Wiesner, R. H., Dahlke, L. J., and Krom, R. A. (1994). FK506Induced Neurotoxicity in Liver Transplantation. Ann. Neurol. 35, 498-501. doi:10.1002/ana.410350422

Zavascki, A. P., Goldani, L. Z., Cao, G., Superti, S. V., Lutz, L., Barth, A. L., et al. (2008). Pharmacokinetics of Intravenous Polymyxin B in Critically Ill Patients. Clin. Infect. Dis. 47 (10), 1298-1304. doi:10.1086/592577

Zavascki, A. P., Goldani, L. Z., Li, J., and Nation, R. L. (2007). Polymyxin B for the Treatment of Multidrug-Resistant Pathogens: A Critical Review. J. Antimicrob. Chemother. 60 (6), 1206-1215. doi:10.1093/jac/dkm357

Conflict of Interest: The authors declare that the research was conducted in the absence of any commercial or financial relationships that could be construed as a potential conflict of interest.

Publisher's Note: All claims expressed in this article are solely those of the authors and do not necessarily represent those of their affiliated organizations, or those of the publisher, the editors and the reviewers. Any product that may be evaluated in this article, or claim that may be made by its manufacturer, is not guaranteed or endorsed by the publisher.

Copyright $\odot 2021$ Li, Deng, Zhu, Liu, Xu, Li, Xie, Yao, Yang, Zhang and Zhou. This is an open-access article distributed under the terms of the Creative Commons Attribution License (CC BY). The use, distribution or reproduction in other forums is permitted, provided the original author(s) and the copyright owner(s) are credited and that the original publication in this journal is cited, in accordance with accepted academic practice. No use, distribution or reproduction is permitted which does not comply with these terms. 